\title{
BMJ Open Relationship between heavy metal exposure and type 2 diabetes: a large- scale retrospective cohort study using occupational health examinations
}

\author{
Jun Ho Ji, ${ }^{1}$ Mi Hyeon Jin, ${ }^{2}$ Jung-Hun Kang, ${ }^{3}$ Soon II Lee, ${ }^{4}$ Suee Lee, ${ }^{5}$ \\ Sung-Hyun Kim, ${ }^{5}$ Sung Yong Oh (D) ${ }^{5}$
}

To cite: Ji JH, Jin MH, Kang J$\mathrm{H}$, et al. Relationship between heavy metal exposure and type 2 diabetes: a large-scale retrospective cohort study using occupational health examinations. BMJ Open 2021;11:e039541. doi:10.1136/ bmjopen-2020-039541

- Prepublication history for this paper is available online. To view these files, please visit the journal online (http://dx.doi. org/10.1136/bmjopen-2020039541).

Received 18 April 2020 Revised 23 January 2021 Accepted 25 January 2021

Check for updates

(C) Author(s) (or their employer(s)) 2021. Re-use permitted under CC BY-NC. No commercial re-use. See rights and permissions. Published by BMJ.

For numbered affiliations see end of article.

Correspondence to

Dr Sung Yong Oh;

drosy@dau.ac.kr

\section{ABSTRACT}

Objectives To investigate the associations between heavy metal exposure and serum ferritin levels, physical measurements and type 2 diabetes mellitus (DM).

Design A retrospective cohort study.

Setting Changwon, the location of this study, is a Korean representative industrial city. Data were obtained from medical check-ups between 2002 and 2018.

Participants A total of 34814 male subjects were included. Of them, 1035 subjects with lead exposure, 200 subjects with cadmium exposure and the 33579 remaining were assigned to cohort $A$, cohort $B$ and the control cohort, respectively. Data including personal history of alcohol and smoking, age, height, weight, the follow-up duration, haemoglobin A1c ( $\mathrm{HbA1c}$ ), fasting blood sugar (FBS), ferritin levels, and lead and cadmium levels within 1 year after exposure were collected.

Primary outcome measure In subjects without diabetes, changes in $\mathrm{FBS}$ and $\mathrm{HbA1C}$ were analysed through repeated tests at intervals of 1 year or longer after the occupational exposure to heavy metals.

Results In Cohort A, DM was diagnosed in 33 subjects. There was a significant difference in lead concentrations between the subjects diagnosed with DM and those without DM during the follow-up period $(3.94 \pm 2.92 \mathrm{mg} /$ $\mathrm{dL}$ vs $2.81 \pm 2.03 \mathrm{mg} / \mathrm{dL}, \mathrm{p}=0.002$ ). Simple exposure to heavy metals (lead and cadmium) was not associated with DM in Cox regression models (lead exposure (HR) $1.01,95 \%$ Cl: 0.58 to $1.77, \mathrm{p} 0.971$; cadmium exposure HR $1.48,95 \%$ Cl: 0.61 to $3.55, p=0.385)$. Annual changes in FBS according to lead concentration at the beginning of exposure showed a positive correlation $(r=0.072$, $\mathrm{p}=0.032$ ).

Conclusion Our findings demonstrated that simple occupational exposure to heavy metals lead and cadmium was not associated with the incidence of DM. However, lead concentrations at the beginning of the exposure might be an indicator of DM and glucose elevations.

\section{INTRODUCTION}

Diabetes mellitus (DM), a common and rising global problem, is one of the leading causes of death, blindness and chronic renal failure. It is also a major risk factor for vascular diseases such as myocardial infarction, stroke
Strengths and limitations of this study

- This study was limited by the single institute data obtained from occupational medical evaluations.

- Another important limitation of the study was the exclusion of females of childbearing age who have decreased serum ferritin due to menstruation.

- This study was a large-scale study to determine the blood concentrations of heavy metals (initial exposure to occupational heavy metal and exposure over a long period of time) and changes in fasting blood sugar and haemoglobin A1c ( $\mathrm{HbA1c}$ ) levels.

- This study showed changes in blood glucose and HbA1c over time after exposure to heavy metals.

and peripheral vascular disease. The increase in social cost due to DM-related morbidity or mortality has intensified efforts to reduce the incidence of DM. The rising incidence of DM is considered to be associated with alterations in lifestyles and other contributing factors, including exposure to several environmental pollutants and industrial chemicals.

With rapid industrial development, exposure to various environmental toxic materials has risen along with DM incidence. Environmental substances that cause endocrine disruption have been defined as endocrinedisrupting chemicals (EDCs) by the U.S. Environmental Protection Agency. ${ }^{1}$ Metals are naturally existing inorganic elements that are present in very small amounts in the body. They are essential for vital processes. Heavy metals are generally defined as metals with relatively high densities, atomic weights or atomic numbers. Heavy metals and metalloids (eg, lead, mercury, cadmium and metalloid arsenic) can affect hormonal activity, suggesting that these compounds are EDCs generally considered to be toxicants. These heavy metals have negative effects on physiology. They might be associated with the 
incidence of DM in some populations. In this study, we particularly focused on the association between exposure to heavy metals and DM. In recent decades, environmental exposure to heavy metals has declined because many countries have begun to pay attention to environmental problems rather than industrial development. However, the unintended exposure to heavy metals in the environment such as older household structures and in drinking water in Flint, Michigan, $\mathrm{USA},{ }^{2}$ is still possible. Such exposure can be due to the illegal, unauthorised disposal of toxic materials including heavy metals from industries. In Korea, occupational exposure to heavy metals is more common than random environmental exposure.

In occupational exposure to heavy metals, relatively few studies have reported whether the degree of exposure has direct or indirect effects on the body or specific diseases. A few population-based studies have focused on the association between metal exposure and diabetes, showing inconsistent results. ${ }^{3-9}$ Most previous studies have examined the association of DM with heavy metal concentrations in the blood or urine at one specific time. ${ }^{67}$

Intense exposure to heavy metals can result in high levels of heavy metals in the blood or urine, whereas light exposure results in extremely low levels. Although longterm, light exposure to heavy metals might only lead to low levels of heavy metals in the blood or urine, heavy metals deposited in organs may be harmful. The deposition of heavy metals in the liver and pancreas can alter gluconeogenesis in the liver and affect insulin secretion, eventually influencing the incidence of DM. Although this study was designed as a retrospective study of long-term occupational exposure to heavy metals (lead and cadmium), instead of measuring the concentration of heavy metals in organs such as the liver, bone and pancreas, the blood concentrations of heavy metals at the beginning of the exposure (within 1 year) were measured and compared with changes in fasting blood sugar (FBS), HbAlc and the incidence of DM in the general population who were not exposed to heavy metals during the same period.

\section{MATERIAL AND METHODS \\ Study population}

Changwon, the location of this study, is a representative industrial city in Korea. Many occupations involve heavy metal exposure, including employees of batterymanufacturing plants. This cohort study was based on the data from occupational health examinations $(n=403253)$ conducted from 2002 to 2018 in subjects with jobs related to heavy metals. A schematic flow chart for the selection of subjects is shown in figure 1. All participants underwent a physical examination with a blood sample taken in the morning following an overnight fast. They also filled out a questionnaire. Among these 403253 subjects, 89826 who had ferritin blood levels measured were included and 38039 women were excluded. In occupational screening, most women were fertile. The ferritin results might be low because of menstruation. A total of 269 subjects were excluded because of the unavailability of HbA1c or FBS data. Furthermore, 2709 subjects who were already diagnosed with DM were excluded (DM was defined as FBS $\geq 126 \mathrm{mg} / \mathrm{dL}, \mathrm{HbA} 1 \mathrm{c} \geq 6.5 \%$, or a history of DM reported in the questionnaire). Additionally, 28151 subjects were excluded because they only had one screening result without follow-up data. Finally, 34814 subjects were included in the analysis. Of these, 1035 subjects with lead exposure, 200 subjects with cadmium exposure and the 33579 remaining subjects were assigned to cohort A, cohort B and the control cohort, respectively. This study

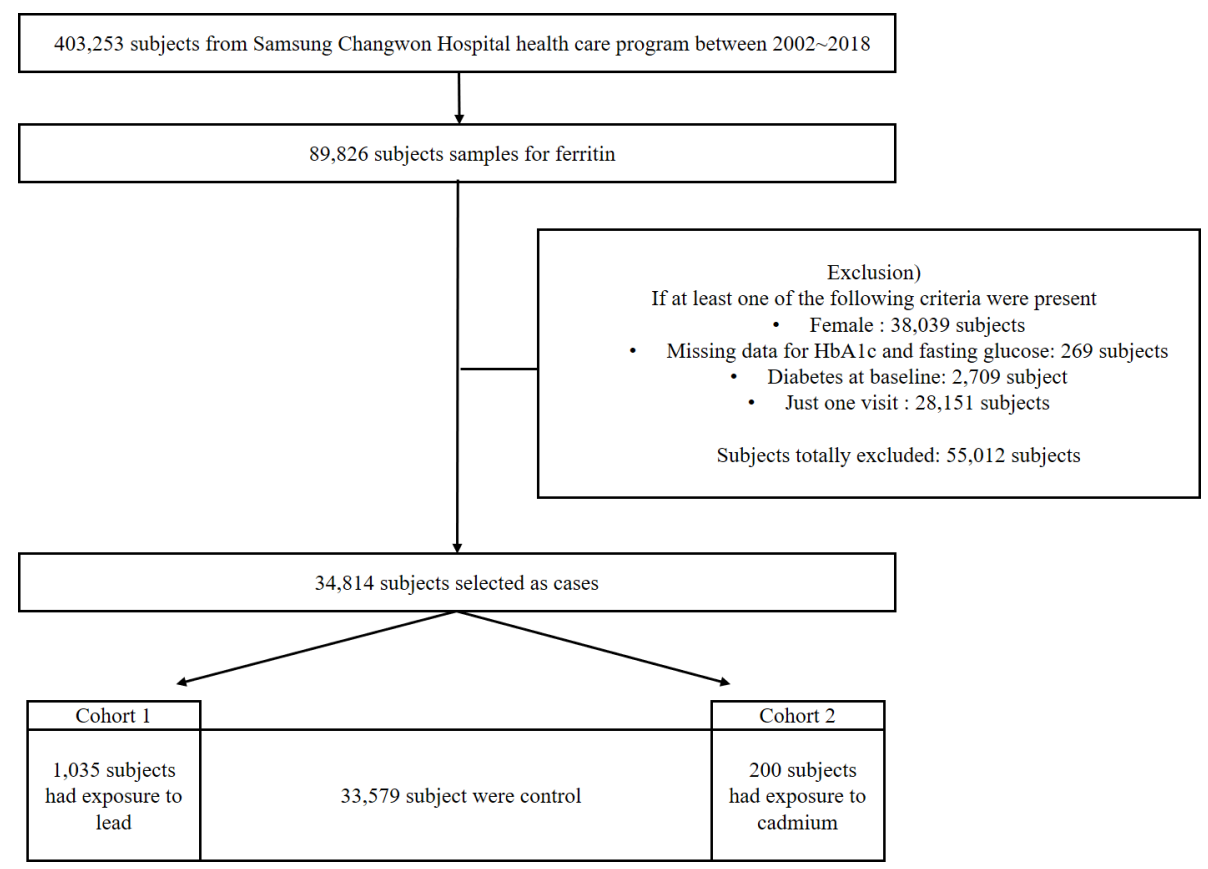

Figure 1 Schematic flow diagram. HbA1c, haemoglobin A1c. 
collected subject data including age, HbA1c, FBS, ferritin levels, height, body weight, the follow-up duration and the concentrations of heavy metals (lead and cadmium)

\section{Data collection}

This study was based on data from occupational health examinations already conducted. The health check-up data included objective numerical data such as blood tests, imaging tests and physical examinations, as well as the questionnaire responses of the subjects. The questionnaire included items on personal history, physical activity, systemic symptoms, sleep patterns, stress, anxiety, depression, gambling and job stress. All data were computerised. The authors analysed the demographic information, physical examination results, history and laboratory results (HbAlc, blood glucose, ferritin, lead and cadmium levels). After obtaining Institutional Review Board approval, two authors (JHJ and $\mathrm{MHJ}$ ) independently analysed the data.

\section{Measuring blood levels of lead and cadmium}

To measure the blood levels of lead and cadmium, $3 \mathrm{~mL}$ of blood was collected from each subject into vacuum bottles using heparin as an anticoagulant in the morning following an overnight fast. Blood samples were diluted 1:15 and 1:10 to measure the lead and cadmium concentrations, respectively, with $2.5 \mathrm{~mL}$ of $10 \%$ Triton X-100, $0.1 \mathrm{~mL}$ of concentrated nitric acid and $1 \mathrm{~mL}$ of $10 \%$ ammonium dihydrogen phosphate as a modifier. Graphite furnace atomic absorption spectrometry with Zeeman background correction (PinAAcle 9i00z Atomic absorption spectrometer, PerkinElmer, Norwalk, Connecticut, USA) was used to measure the lead and cadmium levels in all subjects within the first year of heavy metal exposure. The minimum detectable limits of lead and cadmium were measured to the third decimal place $(0.001 \mathrm{mg} /$ $\mathrm{dL}$ ), and concentrations below that were considered to be zero.

\section{Statistical analyses}

The continuous variables are presented as means \pm SD. The categorical variables are presented as the number of cases and percentages. An independent t-test was used to evaluate the significance of the mean differences between the continuous variables for demographical factors such as age and body mass index (BMI). The Cox proportional hazard model was used to identify potential predictors in the baseline characteristics for type $2 \mathrm{DM}$ in subjects who were not diagnosed with DM. In the Cox hazard model, the development of type 2 DM was considered a dependent variable and as independent variables were set to the exposure levels of lead and cadmium and the known risk factors (age, BMI, smoking, drinking, HbA1c, FBS and ferritin). A mixed model was used to assess the effects of heavy metal exposure and ferritin on FBS and HbAlc, respectively. The annual changes in FBS and HbA1c with lead concentrations are shown in a scatter plot. Stata
V.14.0 software (Stata Corporation, College Station, Texas, USA) was used for all statistical analyses.

\section{Operational definitions}

1. Type $2 \mathrm{DM}$ was defined in patients with a diabetes diagnosis history taking antidiabetic medication or satisfying the American Diabetes Association criteria of HbA1c $\geq 6.5 \%$ or FBS $\geq 126 \mathrm{mg} / \mathrm{dL}$ in a blood test after an 8-hour fast.

2. Newly diagnosed diabetes was defined in subjects without a history of diabetes who had an HbAlc of $<6.5 \%$ and an FBS of $<100 \mathrm{mg} / \mathrm{dL}$ in the first health check-up after joining the company and were newly diagnosed with diabetes (HbA1c $\geq 6.5 \%$ or FBS $\geq 126 \mathrm{mg}$ / $\mathrm{dL}$ ) in a follow-up health check-up conducted at least 1 year later.

3. The heavy metal exposure subjects were those who worked in the lead industry, those who were in charge of lead welding and mounting in shipyards and subjects who worked in Ni-Cd battery manufacturing factories.

4. Simple occupational exposure to lead or cadmium, called simple exposure, referred to subjects who worked on-site at the workplace regardless of the intensity of the exposure.

5. The beginning of exposure referred to the first occupational health examination conducted within a year of working in the workplace related to heavy metal exposure.

\section{Patient and public involvement}

The patients and the public were not involved in the development of the research question or the design of the study. No patients or public members were involved in the recruitment or conduct of the study. Since this study used deidentified results, the authors do not plan to disseminate the study results to the study participants individually but plan to publish the paper with open access.

\section{RESULTS}

\section{Baseline characteristics of the study subjects}

The baseline characteristics of the subjects in each cohort are shown in table 1 . Of 34818 subjects, 1034 were diagnosed with DM during the follow-up and 33780 were not diagnosed with DM. In cohort A (1035 subjects with lead exposure and 33779 subjects with no lead exposure), 1034 were confirmed to have DM. Of these 1034 subjects, 33 were exposed to lead. In the control group without heavy metal exposure, age, HbA1c, FBS and ferritin levels were associated with DM, as expected. In heavy metal-exposed subjects, only HbAlc, FBS and BMI were significantly associated with DM. An interesting aspect in cohort A was that the initial concentration of lead (within 1 year) was significantly higher in subjects who were later diagnosed with DM $(2.81 \pm 2.03 \mathrm{mg} / \mathrm{dL}$ in patients not diagnosed with diabetes and $3.94 \pm 2.92 \mathrm{mg} / \mathrm{dL}$ in patients diagnosed 
Table 1 Baseline characteristics

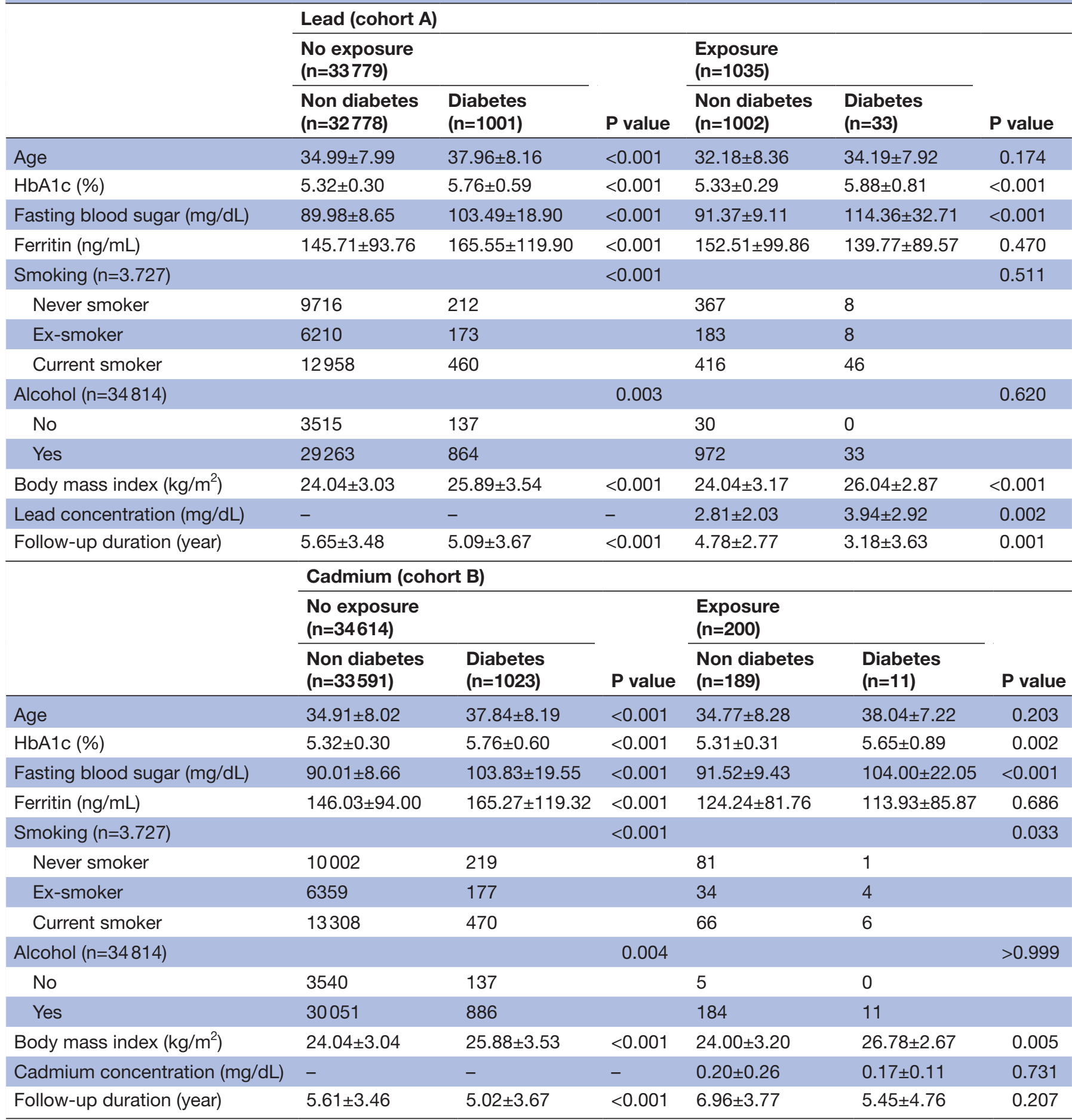

HbA1c, haemoglobin A1c.

with diabetes, $\mathrm{p}=0.002$ ). In contrast, the early cadmium blood levels did not differ between the group of subjects progressing to DM and those who did not progress to DM. The rates of drinking and smoking in patients with diabetes were similar to that in previous studies. Overall, the incidence of diabetes was higher in drinkers than in non-drinkers and higher in smokers than in ex-smokers or never smokers. However, the total number of subjects exposed to heavy metals was small, resulting in no statistical significance. The follow-up period was shorter and the mean age was higher in subjects progressing to DM in both cohorts. In the lead-exposed group, the mean follow-up duration was $3.18 \pm 3.63$ years for the group with $\mathrm{DM}$ and $4.78 \pm 2.77$ years $(\mathrm{p}=0.001)$ for the nondiabetes group. In the cadmium-exposed group, the mean follow-up duration was $5.45 \pm 4.76$ years for the DM 
Table 2 Cox regression models: crude and adjusted HRs of baseline characteristics predicting the development of type 2 diabetes mellitus

\begin{tabular}{|c|c|c|c|c|}
\hline & \multicolumn{2}{|l|}{ Crude } & \multicolumn{2}{|l|}{ Adjusted ( $\mathrm{N}=30589$ ) } \\
\hline & HR $(95 \% \mathrm{Cl})$ & $P$ value & HR $(95 \%$ Cl) & $P$ value \\
\hline Age (year) & 1.05 (1.04 to 1.06$)$ & $<0.001$ & 1.01 (1.00 to 1.03$)$ & 0.012 \\
\hline Haemoglobin A1c (\%)×10 & 1.54 (1.51 to 1.57$)$ & $<0.001$ & 1.35 (1.32 to 1.39$)$ & $<0.001$ \\
\hline Fasting blood sugar (mg/dL) & 1.12 (1.11 to 1.12 ) & $<0.001$ & 1.07 (1.06 to 1.08$)$ & $<0.001$ \\
\hline Body mass index $\left(\mathrm{kg} / \mathrm{m}^{2}\right)$ & 1.21 (1.19 to 1.22$)$ & $<0.001$ & 1.10 (0.078 to 1.12$)$ & $<0.001$ \\
\hline $\begin{array}{l}\text { Ferritin } \\
\text { (ng/mL, reference: <200) }\end{array}$ & 2.25 (1.94 to 2.62) & $<0.001$ & 1.51 (1.28 to 1.79$)$ & $<0.001$ \\
\hline Lead exposure & 1.05 (0.68 to 1.63$)$ & 0.812 & 1.01 (0.58 to 1.77$)$ & 0.971 \\
\hline Cadmium exposure & 1.08 (0.54 to 2.17$)$ & 0.828 & 1.48 (0.61 to 3.55$)$ & 0.385 \\
\hline \multicolumn{5}{|l|}{ Smoking } \\
\hline Ex-smoker & 1.22 (0.98 to 1.51$)$ & 0.071 & 1.05 (0.85 to 1.31$)$ & 0.634 \\
\hline Current smoker & 1.61 (1.35 to 1.92$)$ & $<0.001$ & 1.45 (1.22 to 1.73$)$ & $<0.01$ \\
\hline Drinking & 0.83 (0.68 to 1.01 ) & 0.062 & 1.07 (0.53 to 2.17$)$ & 0.842 \\
\hline
\end{tabular}

group and $6.96 \pm 3.77$ years $(\mathrm{p}=0.207)$ for the non-diabetes group.

\section{Risk of developing DM from lead/cadmium exposure and serum ferritin levels}

The Cox-regression models showed the crude and adjusted hazard ratios of the variables predicting the development of DM (table 2). Age, HbAlc, FBS, BMI, current smoking and ferritin were predictors for developing DM in both the crude and adjusted models. However, simple exposure to lead or cadmium was not associated with DM. Ferritin levels had a positive relationship with FBS and HbA1c elevations during the follow-up period in both cohorts A and B (figures 2A,B and 3A,B). FBS elevations in subjects with simple lead exposure were slower than in those without lead exposure (figure 2C). However, simple lead exposure did not have a significant effect on HbAlc elevation (figure 2D). The association of early cadmium exposure on the FBS/HbA1c change was not different from that of lead. In cohort B, ferritin also had significant effects on the elevation of FBS and HbA1c (figure 3A,B). Early exposure to cadmium was positively correlated with the rate of FBS change but negatively correlated with $\mathrm{HbAlc}$ change (figure 3C,D). The unusual finding in both cohorts was that all subjects were healthy, without DM at the time of enrolment. However, subjects with elevated ferritin and heavy metal exposure had higher baseline FBS and HbA1c values than those who did not (figures 2E,F and 3E,F). Regarding the concentrations of heavy metals, annual variations in FBS according to the initial lead concentrations showed weak but positive correlations $(\mathrm{r}=0.072, \mathrm{p}=0.032$, figure 4$)$.

\section{DISCUSSION}

Many studies have attempted to explain the relationship between heavy metal exposure and hyperglycaemia. Several plausible hypotheses have resulted from such research. First, oxidative stress caused by heavy metals can directly damage beta cells of the pancreas, leading to elevated serum glucose levels. ${ }^{10-17}$ Such oxidative stress may also increase blood glucose levels by decreasing insulin release, impairing insulin receptors, disrupting glucose uptake, increasing hepatic gluconeogenesis and pancreatic glucagon secretion, and decreasing peripheral glucose use. ${ }^{16-22}$ Another hypothesis is related to the competitive inhibition of toxic metals. It states that essential trace metals at normal levels play a key role in glucose homeostasis because these metals are essential cofactors for glucose metabolism, pancreatic beta-cell function and the insulin signalling cascade. ${ }^{18} 192324$ Toxic metals compete with these essential metals for various physiological functions and affect type $2 \mathrm{DM}$ risk. ${ }^{25}{ }^{26} \mathrm{It}$ has also been reported that toxic metals can affect various substances, including glucose transporter type 4, nuclear factor kappa B, mitogen-activated protein kinases and phosphoinositide 3-kinase involved in insulin signalling, thereby increasing the risk of DM. ${ }^{27-31}$ The last hypothesis is that exposure to metals, especially heavy metals, can increase body weight, a theory based on population studies. Because weight gain is a known risk factor for DM, exposure to heavy metals might be associated with DM. ${ }^{32-36}$ Many studies on the relationship between heavy metal exposure and DM have been performed based on these findings. However, they showed inconsistent results. ${ }^{3-9}$ Thus, it can be inferred that a direct association between heavy metals and DM has not yet been confirmed. Even if such association is relevant, it is very weak. The prior epidemiologic studies reporting inconsistent results connecting heavy metals to DM have limitations. Most previous studies had cross-sectional designs. ${ }^{3-5}$ 7-9 $\mathrm{A}$ cross-sectional study is characterised by an analysis conducted at a specific point in time. It does not reflect changes over time. In the case of heavy metal exposure, chronic long-time exposure is more common 
A

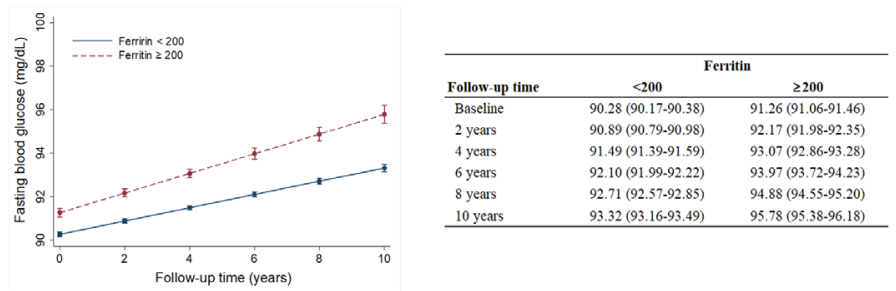

田
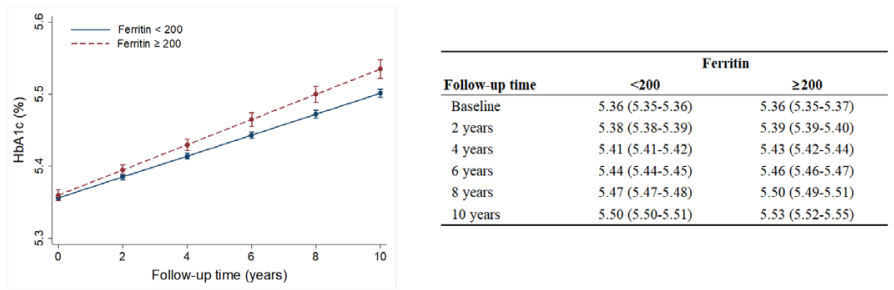

c
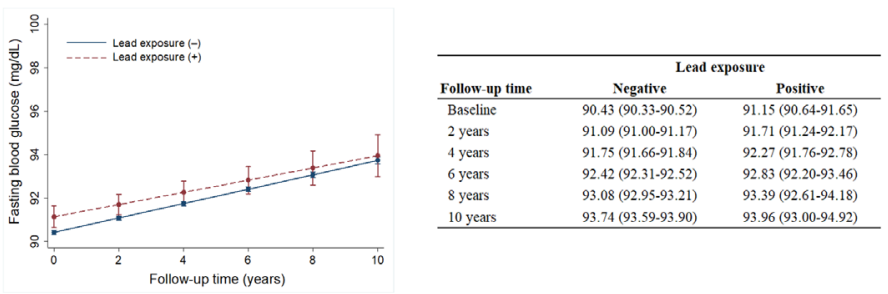

口
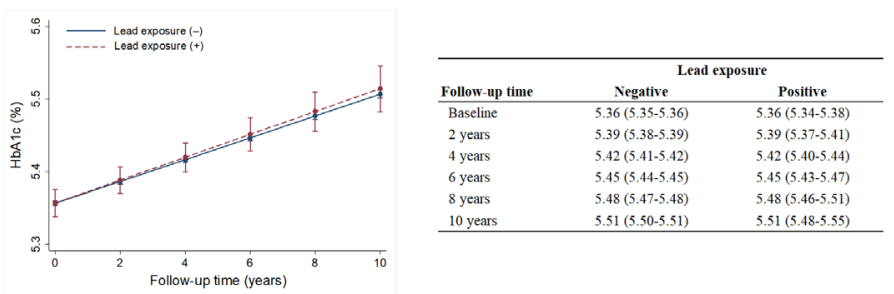

E
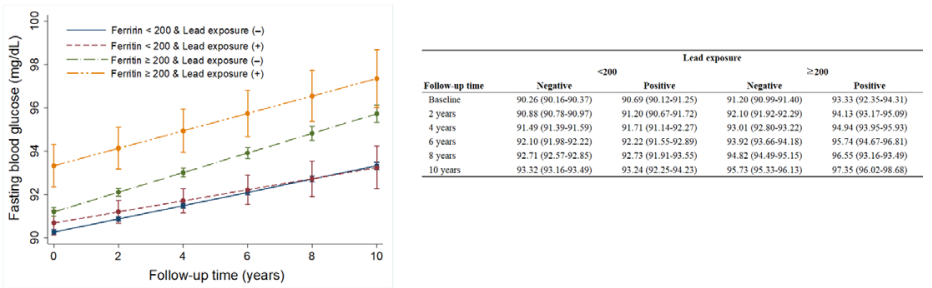

F
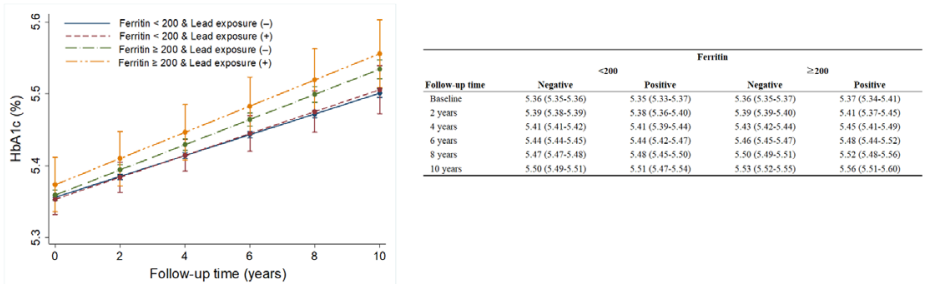

Figure 2 Mixed models were used to evaluate the effects of lead exposure and ferritin on fasting blood sugar and haemoglobin $\mathrm{A} 1 \mathrm{c}(\mathrm{HbA1c})$. (A) Changes in fasting blood glucose according to serum ferritin levels in cohort $\mathrm{A}$. (B) Changes in $\mathrm{HbA1c}$ according to serum ferritin levels in cohort A. (C) Changes in fasting blood glucose according to lead exposure in cohort A. (D) Changes in HbA1c according to lead exposure in cohort A. (E) Changes in fasting blood glucose according to serum ferritin levels and lead exposure in cohort A. (F) Changes in HbA1c according to serum ferritin levels and lead exposure in cohort A. 
A
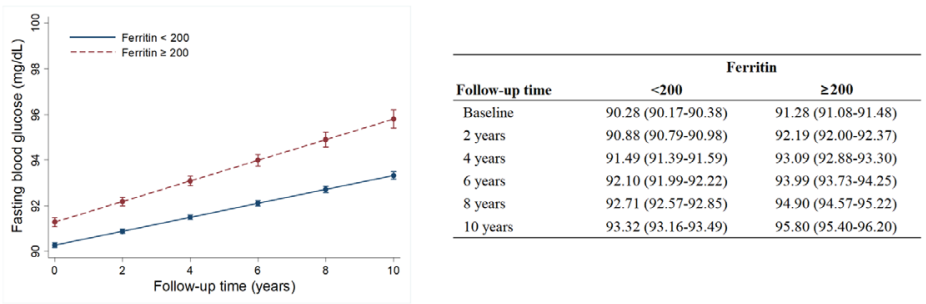

B
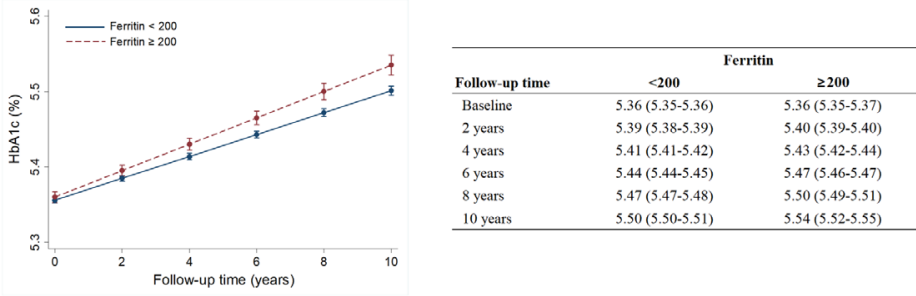

0
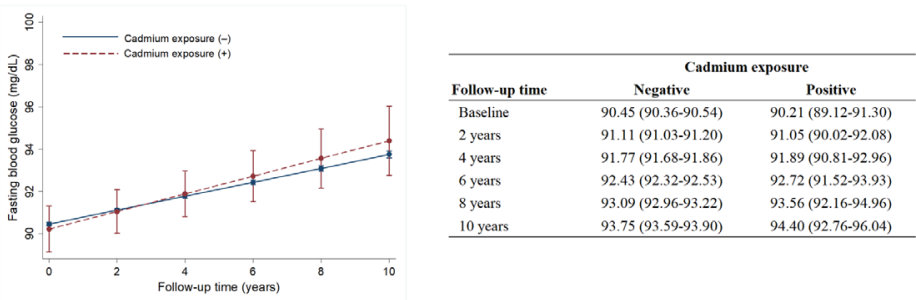

0
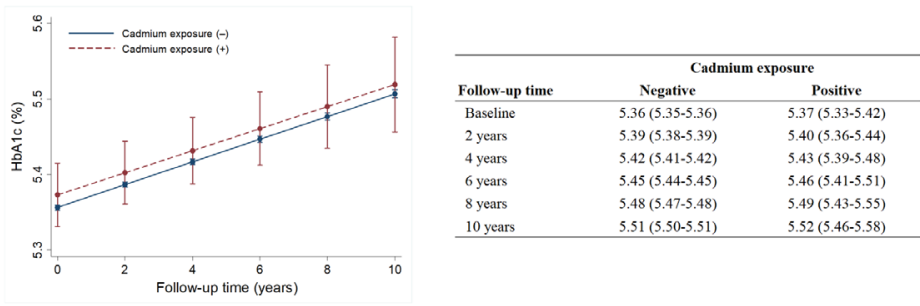

E
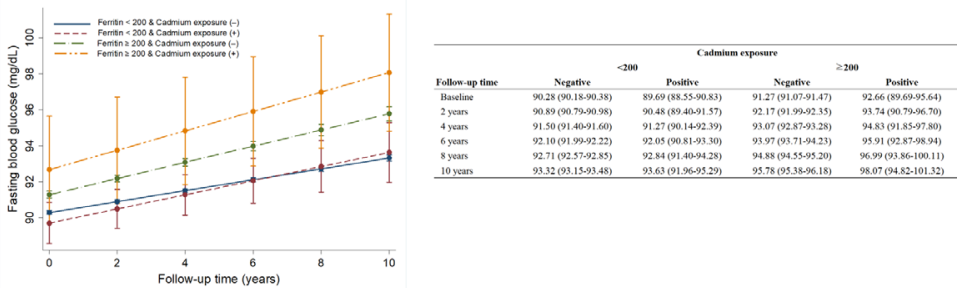

F
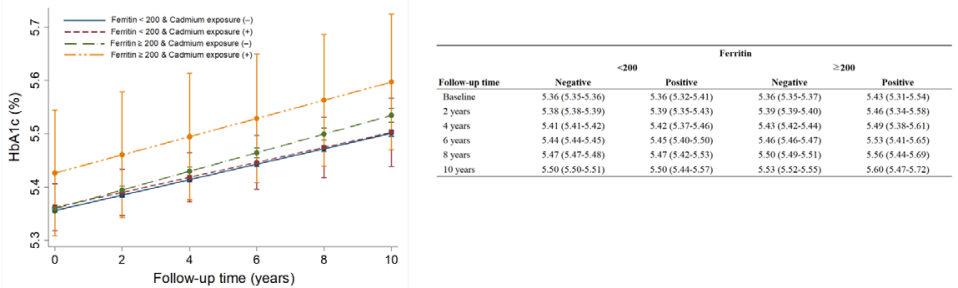

Figure 3 Mixed models were used to evaluate the effects of cadmium exposure and ferritin on fasting blood sugar and haemoglobin $\mathrm{A} 1 \mathrm{c}(\mathrm{HbA1c})$. (A) Changes in fasting blood glucose according to serum ferritin levels in cohort $\mathrm{B}$, (B) Changes in $\mathrm{HbA1c}$ according to serum ferritin levels in cohort $\mathrm{B},(\mathrm{C})$ Changes in fasting blood glucose according to lead exposure in cohort B. (D) Changes in HbA1c according to lead exposure in cohort B. (E) Changes in fasting blood glucose according to serum ferritin levels and lead exposure in cohort B. (F) Changes in HbA1c according to serum ferritin levels and lead exposure in cohort B. 


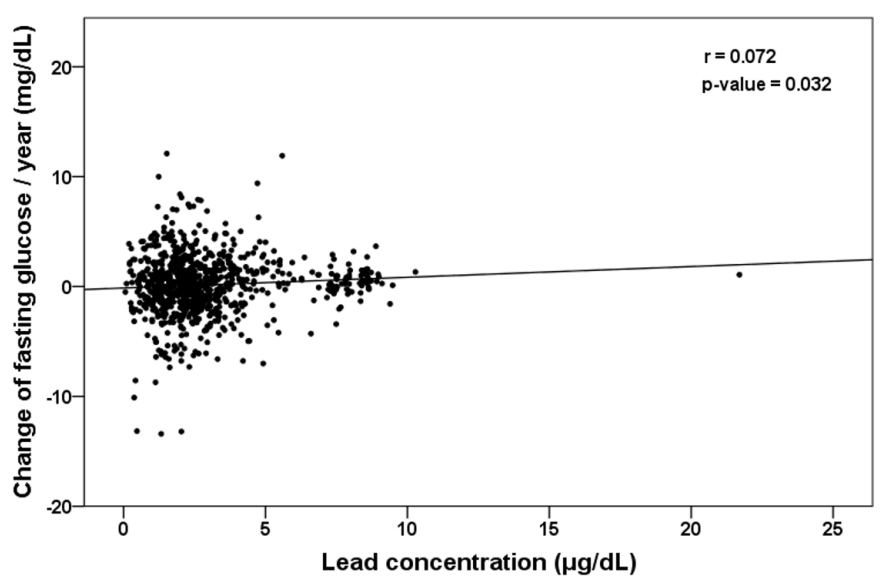

Figure 4 Scatter plot showing the annual changes of fasting blood glucose by lead concentration ( $r=0.072, p=0.032)$.

than acute exposure. Therefore, the time of exposure to heavy metals is important. The time elapsed since the first exposure should be also considered. A Chinese study reported that insulin secretion was decreased more in the group exposed to cadmium for more than 10 years than in the group exposed to cadmium for less than 10 years. ${ }^{37}$ Previous studies have also been conducted with a case-control design. ${ }^{39} 3839$ It is well-known that a small case-control study tends to be less expensive and shorter in duration. However, it has a low level of evidence.

This study investigated the relationships between serum ferritin levels, exposure to heavy metals and DM during the health screening of subjects who worked in battery, paint and bullet manufacturing facilities, shipyards or workplaces requiring welding. Although this study included data from a single institution, it was designed as a retrospective longitudinal study using a large number of health screening subjects, thus overcoming the limitations of prior studies. The following results were obtained. (1) Simple exposure to heavy metals did not increase the risk of developing DM over time; however, the concentration of lead at the time of initial lead exposure was higher in subjects later diagnosed with DM. (2) Serum ferritin was a predictor of DM, as previously reported ${ }^{40}$; however, serum ferritin was not a predictor of DM in subjects exposed to lead or cadmium. (3) A high blood lead concentration at the beginning of the lead exposure was proportional to the rate of increase in FBS per year. It was noteworthy that when the blood lead concentration measured within a year after exposure was high, the rate of FBS increased gradually with time. A high blood lead concentration means that the lead exposure intensity is strong in a short time. Thus, lead exposure intensity might be a risk factor for DM. This aligns with our other study results, in which simple exposure to heavy metals was not related to the incidence of DM or elevations in FBS and HbA1c. The concentrations of heavy metals in our cohort were slightly higher than those in the Korean general adult population in a demographic study on environmental exposure to heavy metals by Kim and Lee. ${ }^{41}$ This suggests that our cohort was occupationally exposed to heavy metals. However, their exposure intensity was not high enough to significantly affect the incidence of DM. Similar to our results, a Korean study demonstrated that low-dose lifetime environmental exposure to lead and cadmium might not affect the incidence of DM. Another interesting aspect of this study is shown in table 1. In the lead-exposed and cadmium-exposed groups, serum ferritin levels in the group with diabetes were significantly higher than those in the non-diabetes group, but not in subjects exposed to lead or cadmium (serum ferritin was lower in the diabetes group). The reason for these results cannot be precisely explained. Oxidative stress caused by the production of free radicals, ${ }^{12-16} 18$ a mechanism by which heavy metals cause DM, might be the mechanism involved in the development of DM. ${ }^{42}{ }^{43}$ Some largescale US studies have shown that high blood levels of persistent organic pollutants (POPs), which are not heavy metals but bio-accumulate as heavy metals with chronic environmental exposure globally, pose an increased risk for DM. ${ }^{44}$ The mechanism by which POPs induce DM is similar to that for DM induced by heavy metals. ${ }^{4546}$ Similar to studies on the associations of heavy metals and DM, studies on the associations of POPs with DM also showed discrepant results. ${ }^{47-49}$

The current findings should be interpreted with caution because of several limitations. Since this study was based on data from subjects undergoing health check-ups, we could not identify or analyse the risk factors of DM, including hypertension, family history and dyslipidaemia. The second limitation was that the blood concentrations of heavy metals were measured only once at the beginning of the exposure. Follow-up observations such as the diagnosis of DM were done longitudinally without reflecting changes in the serum concentrations of heavy metals as in a cross-sectional study. The limited study cohort population was another limitation. Because of possible iron deficiency during menstruation, female subjects were excluded. Due to the nature of the industry dealing with heavy metals, it is a limited study cohort to include only young subjects in the study. Although this study excluded female subjects, it would be interesting to investigate the relationship between occupational heavy metal exposure and diabetes in women. Although menstruation can cause iron deficiency, serum ferritin is associated with the risk of developing diabetes in fertile women. ${ }^{40}$ Thus, further studies with female subjects are warranted.

In conclusion, our findings demonstrated that simple exposure to lead or cadmium was not associated with the prevalence of DM. However, blood lead concentrations at the beginning of exposure might be a predictor of DM development and glucose elevations. Our results suggest that low-dose, chronic occupational exposure to lead or cadmium may not affect the incidence of DM. However, if the exposure intensity is high, screening for DM should be performed. 
Author affiliations

${ }^{1}$ Internal Medicine, Samsung Changwon Hospital, Changwon, Gyeonsangnam-do The Republic of Korea

${ }^{2}$ Biostatistics, Samsung Changwon Hospital, Changwon, Gyeonsangnam-do, The Republic of Korea

${ }^{3}$ Internal Medicine, Gyeongsang National University College of Medicine, Jinju, Gyeongsangnam-do, The Republic of Korea

${ }^{4}$ Internal Medicine, Dankook University College of Medicine, Cheonan,

Chungcheongnam-do, The Republic of Korea

${ }^{5}$ Internal Medicine, Dong-A University Medical Center, Busan, The Republic of Korea

Acknowledgements Thanks to all the patients who participated in this study.

Contributors Conception or design: JHJ. Acquisition, analysis or interpretation of data: JHJ. Drafting the work or revising and final approval of the manuscript: All authors.

Funding This study was supported by Dong-A University Research fund 2020. Competing interests None declared.

Patient consent for publication Not required

Ethics approval The study protocol was approved by the Institutional Review Board of Samsung Changwon Medical Center (SCMC-2019-04-014). All participants provided written informed consent for the use of their data.

Provenance and peer review Not commissioned; externally peer reviewed.

Data availability statement Data are available in a public, open access repository. Extra data can be accessed via the Dryad data repository at http://datadryad.org/ with the doi: 10.5061/dryad.tht76hdz4.

Open access This is an open access article distributed in accordance with the Creative Commons Attribution Non Commercial (CC BY-NC 4.0) license, which permits others to distribute, remix, adapt, build upon this work non-commercially, and license their derivative works on different terms, provided the original work is properly cited, appropriate credit is given, any changes made indicated, and the use is non-commercial. See: http://creativecommons.org/licenses/by-nc/4.0/.

\section{ORCID iD}

Sung Yong Oh http://orcid.org/0000-0002-8321-4113

\section{REFERENCES}

1 Diamanti-Kandarakis E, Bourguignon J-P, Giudice LC, et al. Endocrine-Disrupting chemicals: an endocrine Society scientific statement. Endocr Rev 2009;30:293-342.

2 Hanna-Attisha M, LaChance J, Sadler RC, et al. Elevated blood lead levels in children associated with the Flint drinking water crisis: a spatial analysis of risk and public health response. Am J Public Health 2016;106:283-90.

3 Feng W, Cui X, Liu B, et al. Association of urinary metal profiles with altered glucose levels and diabetes risk: a population-based study in China. PLoS One 2015;10:e0123742.

4 Menke A, Guallar E, Cowie CC. Metals in urine and diabetes in U.S. adults. Diabetes 2016;65:164-71.

5 Barregard L, Bergström G, Fagerberg B. Cadmium exposure in relation to insulin production, insulin sensitivity and type 2 diabetes: a cross-sectional and prospective study in women. Environ Res 2013;121:104-9.

6 Hansen AF, Simić A, Åsvold BO, et al. Trace elements in early phase type 2 diabetes mellitus-A population-based study. The HUNT study in Norway. J Trace Elem Med Biol 2017;40:46-53.

7 Moon S-S. Association of lead, mercury and cadmium with diabetes in the Korean population: the Korea National health and nutrition examination survey (KNHANES) 2009-2010. Diabet Med 2013:30:e143-8.

8 Borné Y, Fagerberg B, Persson M, et al. Cadmium exposure and incidence of diabetes mellitus--results from the Malmö Diet and Cancer study. PLoS One 2014;9:e112277.

9 Forte G, Bocca B, Peruzzu A, et al. Blood metals concentration in type 1 and type 2 diabetics. Biol Trace Elem Res 2013;156:79-90.

10 Gerber PA, Rutter GA. The role of oxidative stress and hypoxia in pancreatic beta-cell dysfunction in diabetes mellitus. Antioxid Redox Signal 2017;26:501-18.

11 Kaneto $\mathrm{H}$, Katakami N, Kawamori D, et al. Involvement of oxidative stress in the pathogenesis of diabetes. Antioxid Redox Signal 2007;9:355-66.
12 Kubisch HM, Wang J, Bray TM, et al. Targeted overexpression of $\mathrm{Cu} / \mathrm{Zn}$ superoxide dismutase protects pancreatic beta-cells against oxidative stress. Diabetes 1997;46:1563-6.

13 Yen C-C, Lu F-J, Huang C-F, et al. The diabetogenic effects of the combination of humic acid and arsenic: in vitro and in vivo studies. Toxicol Lett 2007;172:91-105.

14 Das KK, Das SN, Dhundasi SA. Nickel, its adverse health effects \& oxidative stress. Indian J Med Res 2008;128:412-25.

15 Izquierdo-Vega JA, Soto CA, Sanchez-Peña LC, et al. Diabetogenic effects and pancreatic oxidative damage in rats subchronically exposed to arsenite. Toxicol Lett 2006;160:135-42.

16 Valko M, Morris H, Cronin MTD. Metals, toxicity and oxidative stress. Curr Med Chem 2005;12:1161-208.

17 Kurata Y, Katsuta O, Doi T, et al. Chronic cadmium treatment induces islet B cell injury in ovariectomized cynomolgus monkeys. Jpn J Vet Res 2003;50:175-83.

18 Chen YW, Yang CY, Huang CF, et al. Heavy metals, islet function and diabetes development. Islets 2009;1:169-76.

19 Khan AR, Awan FR. Metals in the pathogenesis of type 2 diabetes. $J$ Diabetes Metab Disord 2014:13:16.

20 Sharma B, Singh S, Siddiqi NJ. Biomedical implications of heavy metals induced imbalances in redox systems. Biomed Res Int 2014:2014:1-26.

21 Beyersmann D, Hartwig A. Carcinogenic metal compounds: recent insight into molecular and cellular mechanisms. Arch Toxicol 2008;82:493-512.

22 Kajimoto $\mathrm{Y}$, Matsuoka $\mathrm{T}$, Kaneto $\mathrm{H}$, et al. Induction of glycation suppresses glucokinase gene expression in HIT-T15 cells. Diabetologia 1999;42:1417-24.

23 Kaur B, Henry J. Micronutrient status in type 2 diabetes: a review. Adv Food Nutr Res 2014;71:55-100.

24 Siddiqui K, Bawazeer N, Joy SS. Variation in macro and trace elements in progression of type 2 diabetes. ScientificWorldJournal 2014;2014:1-9.

25 Ahamed M, Siddiqui MKJ. Environmental lead toxicity and nutritional factors. Clin Nutr 2007;26:400-8.

26 Flora SJS. Structural, chemical and biological aspects of antioxidants for strategies against metal and metalloid exposure. Oxid Med Cell Longev 2009;2:191-206.

27 Walton FS, Harmon AW, Paul DS, et al. Inhibition of insulindependent glucose uptake by trivalent arsenicals: possible mechanism of arsenic-induced diabetes. Toxicol Appl Pharmacol 2004;198:424-33.

28 Han JC, Park SY, Hah BG, et al. Cadmium induces impaired glucose tolerance in rat by down-regulating GLUT4 expression in adipocytes. Arch Biochem Biophys 2003;413:213-20.

29 Somwar R, Koterski S, Sweeney G, et al. A dominant-negative p38 MAPK mutant and novel selective inhibitors of p38 MAPK reduce insulin-stimulated glucose uptake in 3T3-L1 adipocytes without affecting GLUT4 translocation. J Biol Chem 2002;277:50386-95.

30 Souza K, Maddock DA, Zhang Q, et al. Arsenite activation of P13K AKT cell survival pathway is mediated by p38 in cultured human keratinocytes. Mol Med 2001;7:767-72.

31 Zawalich WS, Zawalich KC. A link between insulin resistance and hyperinsulinemia: inhibitors of phosphatidylinositol 3-kinase augment glucose-induced insulin secretion from islets of lean, but not obese, rats. Endocrinology 2000;141:3287-95.

32 Leasure JL, Giddabasappa A, Chaney S, et al. Low-Level human equivalent gestational lead exposure produces sex-specific motor and coordination abnormalities and late-onset obesity in year-old mice. Environ Health Perspect 2008;116:355-61.

33 Faulk C, Barks A, Sánchez BN, et al. Perinatal lead $(\mathrm{Pb})$ exposure results in sex-specific effects on food intake, fat, weight, and insulin response across the murine life-course. PLoS One 2014;9:e104273.

34 Nie X, Wang N, Chen Y, et al. Blood cadmium in Chinese adults and its relationships with diabetes and obesity. Environ Sci Pollut Res Int 2016;23:18714-23

35 Rothenberg SE, Korrick SA, Fayad R. The influence of obesity on blood mercury levels for U.S. non-pregnant adults and children: NHANES 2007-2010. Environ Res 2015;138:173-80.

36 Padilla MA, Elobeid M, Ruden DM, et al. An examination of the association of selected toxic metals with total and central obesity indices: NHANES 99-02. Int J Environ Res Public Health 2010;7:3332-47.

37 Lei L-jian, Jin T-yi, Zhou Y-fen. [The effects of cadmium on the levels of insulin in smelters]. Zhonghua Lao Dong Wei Sheng Zhi Ye Bing Za Zhi 2006;24:3-6.

38 Serdar MA, Bakir F, Hasimi A, et al. Trace and toxic element patterns in nonsmoker patients with noninsulin-dependent diabetes mellitus, impaired glucose tolerance, and fasting glucose. Int J Diabetes Dev Ctries 2009;29:35-40. 
39 Afridi HI, Kazi TG, Brabazon D, et al. Comparative metal distribution in scalp hair of Pakistani and Irish referents and diabetes mellitus patients. Clin Chim Acta 2013;415:207-14.

40 Jiang $\mathrm{R}$, Manson JE, Meigs JB, et al. Body iron stores in relation to risk of type 2 diabetes in apparently healthy women. JAMA 2004;291:711-7.

$41 \mathrm{Kim} \mathrm{N-S}$, Lee B-K. National estimates of blood lead, cadmium, and mercury levels in the Korean General adult population. Int Arch Occup Environ Health 2011;84:53-63.

42 Andrews PA. Disorders of iron metabolism. N Engl J Med 2000;342:1293.

43 Oberley LW. Free radicals and diabetes. Free Radic Biol Med 1988;5:113-24.

44 Lee D-H, Lee I-K, Song K, et al. A strong dose-response relation between serum concentrations of persistent organic pollutants and diabetes: results from the National health and examination survey 1999-2002. Diabetes Care 2006;29:1638-44.
45 Hectors TLM, Vanparys C, van der Ven K, et al. Environmental pollutants and type 2 diabetes: a review of mechanisms that can disrupt beta cell function. Diabetologia 2011;54:1273-90.

46 Enan E, Liu PC, Matsumura F. 2,3,7,8-Tetrachlorodibenzo-P-Dioxin causes reduction of glucose transporting activities in the plasma membranes of adipose tissue and pancreas from the guinea pig. $J$ Biol Chem 1992;267:19785-91.

47 Longnecker MP, Michalek JE. Serum dioxin level in relation to diabetes mellitus among air force veterans with background levels of exposure. Epidemiology 2000;11:44-8.

48 Henriksen GL, Ketchum NS, Michalek JE, et al. Serum dioxin and diabetes mellitus in veterans of operation ranch hand. Epidemiology 1997;8:252-8.

49 Steenland K, Piacitelli L, Deddens J, et al. Cancer, heart disease, and diabetes in workers exposed to 2,3,7,8-tetrachlorodibenzo-p-dioxin. J Natl Cancer Inst 1999;91:779-86. 